\title{
La confiance, pierre angulaire du système éducatif en Finlande
}

Trust, a cornerstone of the education system in Finland

La confianza, piedra angular del sistema educativo en Finlandia

Irmeli Halinen, Hannele Niemi et Auli Toom

Traducteur : Jérôme Quintana

\section{(2) OpenEdition Journals}

Édition électronique

URL : https://journals.openedition.org/ries/5543

DOI : 10.4000/ries.5543

ISSN : 2261-4265

Éditeur

France Education international

Édition imprimée

Date de publication : 1 septembre 2016

Pagination : 147-157

ISBN : 978-2-85420-611-1

ISSN : $1254-4590$

Référence électronique

Irmeli Halinen, Hannele Niemi et Auli Toom, « La confiance, pierre angulaire du système éducatif en Finlande », Revue internationale d'éducation de Sèvres [En ligne], 72 I septembre 2016, mis en ligne le 01 septembre 2018, consulté le 02 juillet 2021. URL : http://journals.openedition.org/ries/5543 ; DOI : https://doi.org/10.4000/ries.5543 


\title{
La confiance, pierre angulaire du système éducatif en Finlande*
}

\author{
Irmeli Halinen \\ Conseil national de l'éducation de Finlande \\ Hannele Niemi \\ Université de Helsinki \\ Auli Toom \\ Université de Helsinki
}

\section{PHILOSOPHIE \\ DE LA CONFIANCE}

Le système éducatif finlandais tel que nous le connaissons aujourd'hui s'est développé de manière cohérente au cours des quarante dernières années. Les principes qui ont guidé sa conception sont fondés sur les notions d'équité dans l'éducation et d'apprentissage tout au long de la vie. Le système vise également à accueillir le plus grand nombre, en ce sens où l'enseignement et l'accompagnement aux études sont dispensés dans les mêmes établissements et dans les mêmes classes à tous les élèves. L'éducation est conçue de manière à fournir des bases solides pour l'apprentissage dès la petite enfance, puis au sein de l'enseignement pré-primaire et de l'enseignement de base. Chacun se verra accompagné dans son apprentissage afin de pouvoir accéder à l'enseignement secondaire et supérieur si la motivation pour entreprendre ce type d'études est suffisamment forte. Le système est souple et aucune voie d'études ne débouche sur une impasse.

La philosophie de la confiance constitue l'une des caractéristiques propres du système éducatif finlandais et se manifeste, dans son fonctionnement même, comme une culture. Outre que l'on fait confiance à chacun dans sa capacité d'apprendre, la confiance se perçoit aussi dans le fonctionnement même du système. Il n'existe guère de mécanismes de contrôle tels que l'inspection ou, pour les élèves, les évaluations à fort enjeu. À l'inverse, tous les changements apportés au système éducatif sont pris en compte ensemble et les efforts entrepris pour développer l'enseignement se font de manière collaborative. Il est

* Article traduit par Jérôme Quintana. 
important que les savoirs et l'apprentissage soient des concepts actifs; les nouveaux savoirs et les nouvelles façons d'appréhender les choses se construisent ensemble. À l'échelle nationale, les évaluations par thème et l'évaluation par échantillonnage des performances des élèves se fondent essentiellement sur le fait de reconnaître la nécessité d'améliorer le système éducatif, non sur le fait de comparer les établissements scolaires entre eux ni sur celui d'imputer l'échec des élèves aux enseignants. L'ensemble du système est dirigé de telle sorte que le but premier soit de veiller à la qualité, tant de manière globale que sur le plan de l'éducation, et de créer les conditions optimales favorisant l'enseignement et l'apprentissage ainsi que le développement et le bien-être de chaque élève.

Cette même philosophie et culture de la confiance apparaît à l'échelle locale (municipale) et aussi dans l'enseignement et l'apprentissage. Les municipalités sont chargées de l'éducation et de la protection de la petite enfance, de l'éducation pré-primaire et de l'éducation de base. Le curriculum national de base de 2014 renforce systématiquement l'implication active des élèves dans l'apprentissage et dans toutes les tâches scolaires. Les enseignants s'efforcent de guider et d'accompagner leurs élèves, encourageant ces derniers à s'appuyer sur leurs propres points forts et leur motivation personnelle pour mieux apprendre. Les enseignants travaillent également davantage qu'auparavant en collaboration avec les parents et d'autres partenaires, afin de créer un environnement plus diversifié pour favoriser l'apprentissage des élèves et le bien-être de l'ensemble de la communauté éducative. La confiance comme principe directeur du système éducatif exige des enseignants capables d'agir de manière autonome, d'assumer leur responsabilité et de collaborer avec d'autres partenaires (Toom et Husu, 2012).

\section{OUTILS DE COOPÉRATION ET PROCESSUS COLLABORATIFS DE CONCEPTION DU CURRICULUM}

En Finlande, l'un des principaux outils permettant de renforcer la confiance et de parvenir à une compréhension commune des besoins en matière d'éducation réside dans l'élaboration du curriculum à l'échelle locale et nationale. Les processus de réforme du curriculum sont ouverts, interactifs et fondés sur une multitude de coopérations possibles entre les différents acteurs. Ils permettent un véritable dialogue et une authentique réflexion sur la mission du système éducatif et sur les qualités qui lui sont nécessaires, ainsi que sur le rôle des établissements scolaires pour favoriser le bien-être humain et bâtir la société de demain. Les nouvelles technologies sont utilisées à cet effet afin de proposer de nouvelles et de meilleures possibilités d'intervention et de coopération entre les différents acteurs (Vahtivuori-Hänninen et al., 2014). 
Le Conseil national finlandais de l'enseignement (FNBE ${ }^{1}$ ) est responsable des processus de réforme du curriculum. Il a pour tâche d'élaborer le curriculum national de base, est doté d'un pouvoir décisionnaire en la matière et est chargé d'accompagner la mise en place des processus d'élaboration du curriculum à l'échelle locale. Environ tous les dix ans, le gouvernement réforme le décret régissant, d'une part, les objectifs nationaux relatifs à l'éducation et, d'autre part, l'affectation des heures d'enseignement. La dernière réforme relative à l'éducation pré-primaire et de base remonte à 2012 : le FNBE a d'abord invité les enseignants, les directeurs d'établissements, les autorités éducatives locales, les formateurs pédagogiques et les chercheurs de tout le pays à prendre part aux divers processus de conception du curriculum national de base. Des dizaines de groupes de travail se sont formés et des centaines d'auditions d'experts ont été organisées pour réfléchir au nouveau curriculum. À trois reprises, au cours de ce processus, des avant-projets de curriculum ont été publiés sur les différents sites du FNBE. Par ailleurs, les municipalités et les établissements scolaires, ainsi que d'autres groupes d'intérêt, ont été invités à faire part de leurs avis. Toute personne intéressée avait la possibilité de prendre connaissance des avant-projets sous forme numérique et d'émettre un avis en retour. Les commentaires étaient ensuite publiés sur les sites web et pris en compte lors de la phase d'affinage du projet. Certaines organisations issues de la société civile ont joué un rôle très actif dans ce processus et ont bien su faire entendre leur voix.

La version définitive du curriculum réformé de l'éducation de base a vu le jour à la fin de l'année 2014. Le 22 décembre 2014, le Conseil national finlandais de l'éducation a défini des règles d'application du curriculum de base (FNBE 2014). Ces règles prévoient que chaque prestataire de service éducatif (l'autorité éducative locale) élabore un curriculum local conforme au curriculum national et à la Loi sur l'éducation de base. Le curriculum local intègre des directives municipales et des ajustements locaux selon les types d'établissements. Ces derniers ont la possibilité de concevoir un curriculum qui corresponde à leurs besoins spécifiques et à leur profil. Les nouveaux curriculums à l'échelle locale doivent être applicables pour la rentrée scolaire de l'automne 2016.

Outre les groupes de travail autour du nouveau curriculum et les systèmes de retours d'avis par voie numérique, le FNBE a également publié une "feuille de route du curriculum » au tout début du processus de réforme. Cette dernière décrivait les différentes phases du processus national de réforme et proposait un certain nombre de mesures propices à l'échelle locale. Elle dressait également une liste des sujets les plus importants méritant débat et réflexion. Grâce à cette feuille de route, chacun a pu appréhender le processus de réforme du curriculum dans son ensemble et anticiper les questions qu'il était le plus important de résoudre, favorisant ainsi une atmosphère de coopération et de confiance.

1. Finnish National Board of Education (FNBE), dont le site est consultable en anglais à l'adresse suivante : http:// www.oph.fi/english $(\mathrm{NdT})$. 
La réforme du curriculum s'est appuyée sur une large et solide base de connaissances. Les retours d'avis provenant des municipalités, des établissements scolaires ainsi que d'autres acteurs ont permis au FNBE de mieux cerner les besoins et les attentes du terrain. De plus, le travail des universitaires dans le domaine de la recherche et du développement, notamment au sein des instituts de formation pédagogique, ainsi que les résultats des évaluations nationales et internationales ou encore les projets de développement, ont été largement utilisés au cours du processus de réforme.

En conséquence, la réforme elle-même et le curriculum national de base ont été accueillis favorablement au sein du monde de l'éducation et de l'ensemble de la société finlandaise. La confiance est un élément clé : les enseignants ont confiance dans le fait que le FNBE est réellement à l'écoute de leurs expériences, de leurs besoins et de leurs idées. De son côté, le FNBE a confiance dans le fait que les enseignants et les autorités éducatives locales font de leur mieux pour élaborer un curriculum local conforme aux directives communes. Les autorités locales jouissant d'une grande autonomie dans leur mission de service éducatif, les municipalités ont la possibilité de concevoir leur propre approche dans la façon de mettre en ouvre le curriculum, qui diffère de celle adoptée par d'autres municipalités. Elles ont non seulement le droit de prendre en compte les caractéristiques locales ainsi que les besoins spécifiques des élèves, mais elles sont aussi tenues de le faire. Cela garantit une certaine liberté professionnelle et une part de créativité dans le système éducatif (Halinen et Holappa, 2013).

\section{LA CONFIANCE DANS L'ENSEIGNEMENT ET L'APPRENTISSAGE}

La réforme du curriculum s'est appuyée sur les nouvelles connaissances issues des recherches sur le cerveau et l'apprentissage. Les expériences émotionnelles positives et la motivation dans l'apprentissage, ainsi que l'interaction et la coopération, sont autant d'éléments dont l'importance a été prise en compte dans le cadre du nouveau curriculum national de base. Dans la conception même de l'apprentissage, on met l'accent sur le rôle actif des élèves et la prise de conscience de leur propre apprentissage comme éléments d'un processus d'apprentissage de qualité, centré sur les objectifs. La confiance constitue également un moteur important dans l'apprentissage.

Le curriculum national de 2014 pour l'enseignement de base présente les établissements scolaires comme étant des communautés d'apprentissage, dans des termes qui reflètent l'esprit des valeurs communes et du concept d'apprentissage comme fondement du curriculum. Les aspects qu'il est important de développer sont les suivants : donner du sens à l'apprentissage, proposer des environnements d'apprentissage authentiques, favoriser le plaisir d'apprendre, 
la participation, le dialogue, l'interaction et la confiance. L'apprentissage est vu comme étant inséparable du développement d'un être et de la construction du bien-être d'une communauté (FNBE, 2014b).

Les principes de confiance et d'accompagnement sont visibles dans la culture de fonctionnement des établissements scolaires et dans la façon dont les élèves s’impliquent dans leur travail. On les perçoit notamment dans les procédures d'évaluation de l'éducation de base. Les enseignants sont chargés d'évaluer leurs élèves et de leur proposer un retour d'évaluation selon diverses modalités, mais aussi d'encourager l'évaluation par les pairs et l'auto-évaluation des élèves. L'objectif de l'évaluation est de guider, de faciliter et de favoriser l'apprentissage. La politique forte d'accueil du plus grand nombre accorde une importance primordiale à l'évaluation formative. Les évaluations sont utilisées pour déterminer le type d'accompagnement dont les élèves ont besoin dans leur apprentissage.

Dans le cadre du curriculum national de base de 2014 (FNBE, 2014), l'accent est mis sur le fait que l'école joue un rôle capital pour favoriser chez l'élève le «concept de soi » (self-concept) et "l'auto-efficacité » (self-efficacy). Le retour d'évaluation de la part de l'enseignant revêt une importance particulière. La diversité des modes d'évaluation, ainsi que la possibilité de proposer à l'élève un retour instructif sur son évaluation, constituent des moyens pédagogiques cruciaux qu'utilise l'enseignant pour accompagner le développement global de l'élève et ses progrès sur le plan de l'apprentissage. Les établissements scolaires sont tenus de développer une culture de l'évaluation dont les caractéristiques principales sont les suivantes :

- favoriser un climat positif, fondé sur le soutien, qui encourage les élèves à faire de leur mieux ;

- mettre en place une façon de travailler fondée sur le dialogue et l'interaction qui favorise l'implication et la participation des élèves ;

- aider les élèves à comprendre leurs propres modes d'apprentissage et rendre leurs progrès visibles tout au long du processus d'apprentissage ;

- proposer une évaluation juste et éthique ;

- encourager la diversité des modes d'évaluation ;

- utiliser les renseignements issus de l'évaluation pour planifier l'enseignement et les autres tâches scolaires.

Dans la plupart des cas, l'évaluation a lieu sous forme d'échanges entre les enseignants et les élèves. Les enseignants doivent s'assurer que les élèves ont un retour susceptible de les guider, de favoriser leur apprentissage et de les renseigner sur leurs progrès et leurs réussites. Les réussites encouragent les élèves à poursuivre leur apprentissage mais les aident aussi à comprendre que les échecs et les réponses incorrectes font partie de l'apprentissage. On les intègre à l'enseignement pour mieux favoriser l'apprentissage tout en respectant les élèves, à qui l'on apprend à émettre des remarques sur leur travail personnel ou collectif et à formuler des commentaires constructifs, qu'ils échangent entre eux et avec 
leurs enseignants. Cela crée les conditions nécessaires au développement de l'autoévaluation des élèves et de l'évaluation par les pairs au cours de leur formation dans le cadre de l'éducation de base (FNBE, 2014).

\section{LE Rôle DES ENSEIGNANTS DANS CETTE CULTURE DE LA CONFIANCE}

Les enseignants jouent un rôle actif et autonome au sein du système éducatif finlandais, qui crée l'espace nécessaire à la responsabilité professionnelle, à la liberté pédagogique et à la créativité. Les enseignants, formés à l'université, participent de manière active aux divers processus de développement à l'échelle de la municipalité, de l'établissement et de la classe. Ils sont également chargés de veiller à l'équité, valeur centrale du système éducatif finlandais (Niemi, 2014 ; Niemi et Isopahkala-Bouret, 2015). Les décisions d'ordre pédagogique que prennent les enseignants ont un impact profond sur l'ensemble de la société.

La carrière enseignante est valorisée en Finlande, et les principales raisons d'être tenté par cette carrière et de vouloir y rester ont trait aux caractéristiques du métier d'enseignant, aux conditions de travail, à l'autonomie en tant que professionnel de l'éducation ainsi qu'au statut enviable de ce métier au sein de la société finlandaise. Malgré les difficultés récentes et les coupes budgétaires en matière de politique éducative dans le pays, le travail des enseignants est encore fortement valorisé et les enseignants, considérés comme étant des professionnels de la pédagogie, bénéficient de la confiance et du respect des Finlandais (Kumpulainen, 2014).

Dans le contexte finlandais, les enseignants jouissent du statut de professionnels dotés d'une large autonomie et d'une grande liberté, mais devant également assumer de vastes responsabilités professionnelles pour mener à bien leur tâche dans la classe auprès des élèves et dans l'établissement avec leurs collègues. Les enseignants sont chargés de déterminer l'organisation du travail scolaire et des pratiques pédagogiques de la classe et de définir de manière collégiale les axes de développement et les innovations pédagogiques.

En tant que professionnels de la pédagogie, ils jouent un rôle actif lors du processus d'élaboration du curriculum à l'échelle nationale et locale et collaborent avec d'autres acteurs impliqués dans les différentes phases du processus (Niemi, 2014). La tâche de l'enseignant consiste toutefois, essentiellement, à assumer son rôle de pédagogue dans les diverses situations d'enseignement et d'apprentissage et à effectuer les choix nécessaires sur ce plan pour favoriser le mieux possible l'apprentissage de élèves dans un cadre à la fois encourageant et visant à intégrer le plus grand nombre.

Les relations entre les enseignants finlandais et leurs élèves sont relativement informelles : les enseignants s'efforcent de traiter leurs élèves de manière égale et de faciliter leur apprentissage, en respectant les objectifs du curriculum 
et en répondant aux besoins des élèves. Il existe entre les enseignants et les élèves des rapports de confiance. Les enseignants sont vus comme des références pédagogiques, faisant autorité en la matière, sans être autoritaires. Ce type de rapport s'appuie sur les principes éducatifs et les valeurs centrales du curriculum national de base en Finlande, mais il s'élabore de manière systémique dans le cadre des activités proposées et des relations qu'entretiennent au quotidien les chefs d'établissement et les enseignants, les enseignants et les parents et, au-delà, dans l'ensemble des échanges qui ont lieu dans la classe. Les chefs d'établissement, eux-mêmes enseignants, sont chargés de diriger leur école, où la structure organisationnelle de direction est relativement peu hiérarchisée car plusieurs mécanismes (qualification des personnels, répartition de la charge de travail, responsabilités diverses, salaires, etc.) contribuent à promouvoir l'égalité entre les enseignants. Ces relations informelles entre les personnels, fondées sur l'égalité, ont une influence sur la classe et sur les échanges entre les enseignants et les élèves (Hargreaves et Fullan, 2012).

Les enseignants finlandais s'impliquent de manière active dans divers axes de développement pédagogique à l'échelle nationale et municipale, ainsi qu'à celle de l'établissement et de la classe. Les enseignants s'intègrent au processus de par leurs fonctions, mais ces questions sont également abordées dans le cadre de la formation initiale. Au cours de leurs études, les futurs enseignants acquièrent des connaissances théoriques et pratiques en matière de curriculum, d'élaboration du curriculum et de processus éducatifs. Ils apprennent aussi à mettre en place des environnements d'apprentissage et des pédagogies efficaces. Ils perçoivent également l'importance de la «capacité d'agir » (agency) au sein de la communauté éducative et lors des interactions en classe (Soini et al., 2015). Ils apprennent néanmoins de nombreux aspects importants du travail de l'enseignant une fois entrés dans le métier, lorsqu'ils intègrent la communauté éducative et qu'ils sont confrontés au travail quotidien.

\section{LA CONFIANCE DANS LA FORMATION ENSEIGNANTE}

Tous les enseignants du primaire et du secondaire suivent une formation à l'université depuis les années 1970. Selon les décrets relatifs à la formation des enseignants datant de 1995 et 2005, la certification des enseignants passe par l'obtention d'un BA (bachelor) 180 et d'un MA (master) 120, soit au total 300 crédits ECTS [système européen d'unités capitalisables transférables], sachant qu'un crédit ECTS équivaut à une charge de travail d'environ 27 heures. Cela signifie que tous les enseignants doivent obtenir un diplôme de master pour exercer leur métier. La formation enseignante en Finlande se fonde ainsi sur l'acquisition d'un bagage universitaire solide, tout en mettant l'accent sur une approche pratique du métier et en veillant au respect des règles éthiques dans l'exercice de cette profession. 
L'une des grandes réussites en matière de formation des enseignants réside dans le fait que le pays a su attirer au sein de la profession des étudiants très doués et motivés. Les cursus de formation pédagogique sont parmi les plus demandés dans les universités finlandaises. Au cours des vingt dernières années, les départements de formation des enseignants ont accueilli d'excellents candidats à la profession. La sélection est très forte : les programmes de formation pour l'enseignement de base (class teacher education) n'intègrent que 5 à $10 \%$ des candidats dont le profil est jugé de qualité ; pour les programmes de formation combinant enseignement de base et enseignement secondaire et/ou adulte (subject teacher education) $)^{2}$, ce taux varie de 20 à $40 \%$ selon les disciplines (Kumpulainen, 2014).

En Finlande, la politique nationale d'évaluation (dont l'assurance qualité fait partie intégrante) repose sur la notion de progrès, à savoir que l'évaluation constitue un outil au service de l'amélioration. C'est un principe fondamental qui s'applique aussi à l'ensemble de l'enseignement supérieur et à la formation des enseignants. Dans l'enseignement supérieur finlandais, il n'existe pas de procédure d'accréditation. Les universités qui détiennent le droit de décerner des diplômes sont responsables de la qualité de ces derniers.

Dans l'enseignement supérieur finlandais, il existe trois niveaux d'assurance qualité (Niemi et Lavonen, 2012) : (1) au plan national, des audits ainsi que d'autres modalités d'évaluation externes. L'assurance qualité dans le cadre de la formation des enseignants repose sur le cadre commun national relatif à l'enseignement supérieur ; (2) au plan institutionnel, la responsabilité en termes de qualité de la formation enseignante incombe principalement aux universités. Ainsi, la formation des enseignants fait partie intégrante de la politique propre à chaque université en matière d'assurance qualité. Les universités établissent leurs propres méthodes pour garantir l'assurance qualité et les programmes de formation des enseignants suivent les directives données; (3) enfin, à l'échelle du département et du programme de formation, il existe des processus impliquant les acteurs du terrain visant à améliorer la qualité de l'apprentissage des élèves. Ces méthodes d'assurance qualité permettent d'être au plus près des élèves et des enseignants en formation.

Les universités possédant un haut degré d'autonomie pour concevoir leur curriculum, il n'existe pas de curriculum détaillé de formation des enseignants valable pour toutes les universités de Finlande. Si tous les établissements chargés d'assurer la formation des enseignants se conforment aux directives et aux lignes directrices en matière de compétences requises pour la certification des enseignants, ils s'appuient néanmoins aussi sur leurs propres caractéristiques pour la mise en œuvre du curriculum.

2. Pour de plus amples informations sur la distinction entre class teacher education et subject teacher education en Finlande, voir (en anglais) : http://www.helsinki.fi/teachereducation/education/classteacher/ (NdT). 
La Finlande, contrairement à de nombreux autres pays, n'a pas emprunté la voie de la standardisation, qui consiste à harmoniser les programmes de formation pédagogique, à proposer une évaluation centralisée de cette formation et à adopter une forte politique d'assurance qualité au plan national. Le pays a plutôt mis l'accent sur l'importance des différents niveaux dans le processus d'assurance qualité et sur leur autonomie. Par exemple, au niveau du département universitaire, l'assurance qualité consiste principalement à permettre de manière continue et collaborative l'élaboration de programmes de formation des enseignants. La Finlande aborde la notion de «qualité » de manière globale : la qualité de l'éducation conduit aux acquis attendus de l'apprentissage, tels qu'ils apparaissent dans les prescriptions nationales et dans le curriculum élaboré à l'échelle du département et du programme, dans des contextes donnés.

Il n'existe en Finlande ni conseil des enseignants (teachers' council) ni agence externe chargée de décerner un certificat ou une licence d'enseignement après l'obtention du diplôme universitaire. Le décret sur la qualification des enseignants (décret gouvernemental 794/2004) définit les compétences requises pour enseigner dans les divers niveaux ou secteurs du système éducatif. Les apprentis-enseignants sont libres de choisir différents cours, selon leur projet professionnel. Lorsqu'elles recrutent un nouvel enseignant, les autorités éducatives locales (ou parfois les chefs d'établissement) s'assurent que les postulants possèdent la formation adéquate, conforme à leur diplôme.

Le modèle finlandais repose sur la confiance : tous les acteurs impliqués dans la formation pédagogique veillent à ce que leurs différents processus maintiennent un haut degré d'efficacité. Jusqu'à présent, ce système a bien fonctionné puisqu'à tous les niveaux de l'assurance qualité, les responsabilités sont assumées avec sérieux. Cela veut également dire qu'il faut veiller à un échange constant entre les différents acteurs. La confiance ne se satisfait pas du statu quo : elle demande un travail permanent.

Si les enseignants finlandais participent pour la plupart de manière active à la formation continue, il faudrait les encourager à s'engager plus souvent encore dans des activités de développement professionnel à travers une offre de formation continue proposée plus systématiquement. Si l'on aidait les enseignants en formation initiale et continue à réguler la classe de manière à favoriser l'apprentissage des élèves, à concevoir des environnements d'apprentissage plus efficaces, à utiliser des méthodes pédagogiques qui mettent les élèves en action, à recourir à des pratiques collégiales et de coopération, enfin, à adopter des supports numériques aux usages multiples, le bien-être des élèves et des enseignants à l'école s'en trouverait amélioré.

La culture de la confiance et de la coopération est présente à tous les niveaux du système éducatif finlandais, de l'enseignement préscolaire aux études supérieures. Résultat: tout le monde a les mêmes chances face aux études et 
c'est le développement global des élèves que l'on valorise. Autres éléments perceptibles de cette culture : les élèves finlandais obtiennent des scores très élevés lors des évaluations internationales de type PISA et les différences entre les établissements scolaires sont minimes (OCDE, 2016). Cette culture a par ailleurs permis de susciter l'attrait des jeunes pour la profession et de valoriser le métier d'enseignant.

De nombreux défis se posent toutefois, dans la société finlandaise actuelle, susceptibles de mettre en péril cette philosophie de la confiance. De substantielles coupes budgétaires ces dernières années, dans le domaine de l'éducation, pourraient, selon les municipalités, accroître des différences de qualité entre les établissements. Ces coupes ont par ailleurs un impact sur le bien-être des élèves et des enseignants à l'école. Le principe de l'accueil de tous les élèves dans l'éducation de base exige des moyens permettant aux enseignants d'aider tous les types d'apprenants, dans leur diversité. Cela implique d'avoir un personnel en nombre suffisant, des environnements d'apprentissage adéquats et de bons supports pédagogiques. Aujourd'hui, le taux de décrochage dans l'éducation de base est de moins de $1 \%$, mais si celui-ci augmente, il génèrera une certaine défiance à l'égard du système éducatif.

De nouveaux défis positifs surgissent, liés à la mise en œuvre du curriculum national de base de 2014. Faire de l'école une communauté d'apprentissage, renforcer le rôle actif des élèves et la coopération entre les enseignants et les divers acteurs, voilà les nouveaux défis dans le domaine de l'apprentissage auxquels tous les enseignants sont confrontés. Les enseignants doivent travailler de façon plus transversale et bâtir ensemble, ainsi qu'avec les élèves, des modules d'apprentissage favorisant une approche globale, fondés sur le projet. Il faudrait développer les modalités de formation continue des enseignants à partir des besoins identifiés plus haut et proposer ainsi un parcours systématique de formation professionnelle.

\section{BIBLIOGRAPHIE}

FNBE (2014): National Core Curriculum for Basic Education 2014, Helsinki: The Finnish National Board of Education.

HALINEN I., HOLAPPA A-S. (2013) : "Curricular balance based on dialogue, cooperation and trust - The case of Finland ", dans W. KUIPER et J. BERKVENS (dir.), Balancing curriculum regulation and freedom across Europe. CIDREE Yearbook 2013, p. 39-62, Enschede : SLO Netherlands Institute for Curriculum Development.

HARGREAVES A., FULLAN M. (2012) : Professional capital: Transforming teaching in every school, New York: Teachers College Press and Toronto : Ontario Principles' Council.

KUMPULAINENT T. (dir.) (2014) : Opettajat Suomessa 2013. [Teachers in Finland], Follow-up report 2014, Helsinki : Finnish National Board of Education [en finnois]. 
NIEMI H. (2014) : "Purposeful Policy and Practice for Equity and Quality - a Finnish Case ", dans Sing Kong LEE, Wing On LEE, Ee Ling LOW (dir.), Education Policy Innovations: Levelling Up and Sustaining Educational Achievement, Singapore: Springer, p. 103-121.

NIEMI H., ISOPAHKALA-BOURET U. (2015) : «Persistent Work for Equity and Lifelong Learning in the Finnish Educational System », The New Educator, p. 130-145.

NIEMI H., LAVONEN J. (2012) : «Evaluation for improvements in Finnish Teacher Education " dans HARFORD J., HUDSON B. et NIEMI H. (dir.), Quality Assurance and Teacher Education: International Challenges and Expectations, vol. 6., Oxford: Peter Lang, p. 159-186.

OECD (2016) : The Programme for International Student Assessment (PISA), https:// www.oecd.org/pisa/aboutpisa/

SOINI T., PYHÄLTÖ K., PIETARINEN J. (2010) : Pedagogical well-being: reflecting learning and well-being in teachers' work. Teachers and Teaching: Theory and Practice, 16(6), p. 735-751, DOI : 10.1080/13540602.2010.517690

SOINI T., PIETARINEN J., TOOM A., PYHÄLTÖ K. (2015) : What contributes to first year student teachers' sense of professional agency in the classroom? Teachers and Teaching: Theory and Practice, 21(6), p. 641-659. DOI : http://dx.doi.org/ $10.1080 / 13540602.2015 .1044326$

TOOM A., HUSU J. (2012) : "Finnish teachers as "makers of the many": Balancing between broad pedagogical freedom and responsibility", dans H. NIEMI, A. TOOM et A. KALLIONIEMI (dir.), Miracle of Education: The principles and practices of teaching and learning in Finnish schools, p. 39-54, Rotterdam/Boston/Taipei : Sense Publishers.

VAHTIVUORI-HÄNNINEN S., HALINEN I., NIEMI H., LAVONEN J. et LIPPONEN L. (2014): "A New Finnish National Core Curriculum for Basic Education (2014) and technology as an integrated tool for learning ", dans H. NIEMI et al. (dir.), Finnish Innovations et Technologies in Schools, p. 21-32, Sense Publishers. 
